\title{
Spectrum of the strange hidden charm molecular pentaquarks in chiral effective field theory
}

\author{
Bo Wang $\odot,{ }^{1,2, *}$ Lu Meng $\odot,{ }^{2, \dagger}$ and Shi-Lin Zhu ${ }^{2,1, \ddagger}$ \\ ${ }^{1}$ Center of High Energy Physics, Peking University, Beijing 100871, China \\ ${ }^{2}$ School of Physics and State Key Laboratory of Nuclear Physics and Technology, \\ Peking University, Beijing 100871, China
}

(Received 31 December 2019; accepted 3 February 2020; published 18 February 2020)

\begin{abstract}
We calculate the effective potentials of the $\Xi_{c} \bar{D}^{(*)}, \Xi_{c}^{\prime} \bar{D}^{(*)}$, and $\Xi_{c}^{*} \bar{D}^{(*)}$ system s with the chiral effective field theory up to the next-to-leading order. We simultaneously consider the short-, intermediate-, and longrange interactions. With the newly observed $P_{c}$ spectra as inputs, we construct the quark-level contact Lagrangians to relate the low energy constants to those of $\Sigma_{c} \bar{D}^{(*)}$ with the help of the quark model. Our calculation indicates there are seven bound states in the $I=0$ strange hidden charm $\left[\Xi_{c}^{\prime} \bar{D}^{(*)}\right]_{J}\left(J=\frac{1}{2}, \frac{3}{2}\right)$ and $\left[\Xi_{c}^{*} \bar{D}^{(*)}\right]_{J}\left(J=\frac{1}{2}, \frac{3}{2}, \frac{5}{2}\right)$ systems. Our analyses also disfavor the $\Lambda_{c} \bar{D}^{(*)}$ bound states. However, we obtain three new hadronic molecules in the isoscalar $\left[\Xi_{c} \bar{D}^{(*)}\right]_{J}\left(J=\frac{1}{2}, \frac{3}{2}\right)$ systems. The masses of $\left[\Xi_{c} \bar{D}\right]_{1 / 2}$, $\left[\Xi_{c} \bar{D}^{*}\right]_{1 / 2}$, and $\left[\Xi_{c} \bar{D}^{*}\right]_{3 / 2}$ are predicted to be $4319.4_{-3.0}^{+2.8} \mathrm{MeV}, 4456.9_{-3.3}^{+3.2} \mathrm{MeV}$, and $4463.0_{-3.0}^{+2.8} \mathrm{MeV}$, respectively. We also notice the one-eta-exchange influence is rather feeble. Binding solutions in the $I=1$ channels are nonexistent. We hope the future analyses at $\mathrm{LHCb}$ can seek for these new $P_{c s} \mathrm{~s}$ in the $J \psi \Lambda$ final states, especially near the thresholds of $\Xi_{c} \bar{D}^{(*)}$.
\end{abstract}

DOI: $10.1103 /$ PhysRevD.101.034018

\section{INTRODUCTION}

In the past decades, the renaissance of hadron physics was witnessed. Mesons and baryons, which have the internal configurations $q \bar{q}$ and $q q q$ respectively, have been extensively studied with lattice QCD and various QCD inspired models. The abundant conventional hadrons in the Reviews of Particle Physics [1] reflect the great victory of the quark model. Other more complicated quark configurations, such as $q q \bar{q} \bar{q}, q \bar{q} q q q$, and $q q q \bar{q} \bar{q} \bar{q}$, etc., are not forbidden by QCD. Thus hunting for these type states is a long standing problem for theorists and experimenters. $X(3872)$ is the poster child that opened a new era for hadron physics [2]. After that, more and more $X Y Z$ states were discovered. The multiquark matter becomes one of the hottest topics in recent years [3-8].

Very recently, the LHCb Collaboration reported the observation of three pentaquark states $P_{c}(4312), P_{c}(4440)$, and $P_{c}(4457)$ [9]. Their masses lie several to tens $\mathrm{MeV}$ below the $\Sigma_{c} \bar{D}$ and $\Sigma_{c} \bar{D}^{*}$ thresholds, thus the molecular

\footnotetext{
*bo-wang@pku.edu.cn

†'lmeng@pku.edu.cn

*zhusl@pku.edu.cn
}

Published by the American Physical Society under the terms of the Creative Commons Attribution 4.0 International license. Further distribution of this work must maintain attribution to the author(s) and the published article's title, journal citation, and DOI. Funded by SCOAP ${ }^{3}$. explanation is naturally proposed in many works [10-21]. The $J^{P}$ quantum numbers are undetermined yet, but the theoretically favored ones in the molecular scenario for these three $P_{c} \mathrm{~s}$ are $\frac{1}{2}^{-}, \frac{1}{2}^{-}$and $\frac{3}{2}^{-}$, respectively. Therefore, if the forthcoming measurements for the $J^{P} \mathrm{~S}$ indeed meet the predictions from the molecular pictures, which would give more robust support for the molecular interpretations.

The $X Y Z$ and $P_{c}$ states are the candidates of the hiddencharm multiquark states with inner quark components $Q \bar{Q} q \bar{q}$ and $Q \bar{Q} q q q$, respectively. As indicated in Refs. [22-24], it seems the heavy quark core plays an important role in stabilizing the exotic clusters. This is indeed the case in the atomic physics. For example, the hydrogen molecule consists of two protons and two electrons, which stably exists in the nature. In the hadronic molecular scenario, the interaction between two color singlets (e.g., $\Sigma_{c}$ and $\bar{D}$ ) is very similar to that between electroneutral atoms (e.g., $H$ and $H$ ). The "covalent bond" in the former is attributed to the residual strong interactions, which is equivalently described by the pion-exchange in chiral effective theory or the mesonexchange (e.g., $\pi, \rho, \sigma, \ldots$ ) in the one-boson-exchange model. Therefore, one could actually anticipate the existence of more hadronic molecules in the charmed baryon-anticharmed meson systems when the flavor symmetry group is enlarged to $\mathrm{SU}(3)$.

Starting from the deuteron (an $I=0$ loosely bound $n p$ molecule), one can notice that the interactions between two heavy matter fields tend to form the bound states in the 
lowest isospin channels. $X(3872)$ is another example, which is a good candidate of the $D^{0} \bar{D}^{* 0}$ molecule with isospin $I=0$. The newly reported $P_{c} \mathrm{~s}$ are widely accepted as the $\Sigma_{c} \bar{D}^{(*)}$ bound states with isospins $I=\frac{1}{2}$. Some investigations on the $D D^{*}$ and $\bar{B}^{(*)} \bar{B}^{(*)}$ systems also demonstrated the existence of bound states in $I=0$ channels $[25,26]$.

In our previous work [11], we have systematically investigated the interactions of the $\Sigma_{c} \bar{D}, \Sigma_{c} \bar{D}^{*}, \Sigma_{c}^{*} \bar{D}$, and $\Sigma_{c}^{*} \bar{D}^{*}$ systems in chiral effective field theory. We simultaneously reproduced the newly observed three $P_{c}$ s as the $I=$ $\frac{1}{2}$ hidden-charm $\Sigma_{c} \bar{D}$ and $\Sigma_{c} \bar{D}^{*}$ molecules by introducing the $\Lambda_{c}$ contribution in the two-pion-exchange loop diagrams. In this work, we extend our study to the $\Xi_{c} \bar{D}^{(*)}, \Xi_{c}^{\prime} \bar{D}^{(*)}$, and $\Xi_{c}^{*} \bar{D}^{(*)}$ systems to see whether there exist the bound states in the lowest isospin, i.e., $I=0$ channels. Likewise, these strange hidden charm molecular states might be reconstructed in the $J / \psi \Lambda$ channel at the LHCb experiment. Some investigations suggest searching for these states in the decay modes $\Lambda_{b}\left(\Xi_{b}\right) \rightarrow J / \psi \Lambda K(\eta)$ [27-29].

Based on Ref. [11], we further study the effective potentials of six systems, i.e., $\Xi_{c} \bar{D}^{(*)}, \Xi_{c}^{\prime} \bar{D}^{(*)}$, and $\Xi_{c}^{*} \bar{D}^{(*)}$. They all contain one strange quark. The short-range contact interaction, long-range one-pion-exchange contribution and intermediate-range two-pion-exchange diagrams are all included in the framework of chiral effective field theory (For the reviews of chiral effective field theory, we refer to [30-34]). Considering the hadronic molecules are shallowly bound states, the strange quark dynamics are freezed in the present calculations, which contribution is partially involved in the contact terms. We ignore the $\eta$ and $K$ meson contributions in the loops. The low energy constants (LECs) are well determined by fitting the $P_{c}$ spectra. In this way, we predict the possible strange hidden charm molecular pentaquarks.

This paper is organized as follows. In Sec. II, we give the effective Lagrangians constructed with the chiral and heavy quark symmetries. In Sec. III, we show the expressions of effective potentials. In Sec. IV, we give the numerical results and discussions. In Sec. V, we conclude this work with a short summary. In Appendix A, we bridge the LECs to those of $\Sigma_{c}^{(*)} \bar{D}^{(*)}$ with quark model.

\section{EFFECTIVE LAGRANGIANS WITH THE CHIRAL AND HEAVY QUARK SYMMETRIES}

The effective Lagrangians can be classified as the pion and contact interactions, respectively. We first show the Lagrangians of the charmed baryon (anticharmed mesons) and light pseudoscalar meson interaction. For the charmed baryons, the matrix forms of the spin $-\frac{1}{2}$ antitriplet, spin $-\frac{1}{2}$, and spin- $-\frac{3}{2}$ sextets in the $\mathrm{SU}(3)$ flavor space are expressed as

$$
\mathcal{B}_{\overline{3}}=\left[\begin{array}{ccc}
0 & \Lambda_{c}^{+} & \Xi_{c}^{+} \\
-\Lambda_{c}^{+} & 0 & \Xi_{c}^{0} \\
-\Xi_{c}^{+} & -\Xi_{c}^{0} & 0
\end{array}\right], \quad \mathcal{B}_{6}=\left[\begin{array}{ccc}
\Sigma_{c}^{++} & \frac{\Sigma_{c}^{+}}{\sqrt{2}} & \frac{\Xi_{c}^{\prime+}}{\sqrt{2}} \\
\frac{\Sigma_{c}^{+}}{\sqrt{2}} & \Sigma_{c}^{0} & \frac{\Xi_{c}^{\prime 0}}{\sqrt{2}} \\
\frac{\Xi_{c}^{\prime+}}{\sqrt{2}} & \frac{\Xi_{c}^{\prime 0}}{\sqrt{2}} & \Omega_{c}^{0}
\end{array}\right], \quad \mathcal{B}_{6}^{* \mu}=\left[\begin{array}{ccc}
\Sigma_{c}^{*++} & \frac{\Sigma_{c}^{*+}}{\sqrt{2}} & \Xi_{c}^{*+} \\
\frac{\Sigma_{c}^{*}}{\sqrt{2}} & \Sigma_{c}^{* 0} & \frac{\Xi_{c}^{* 0}}{\sqrt{2}} \\
\frac{\Xi_{c}^{*+}}{\sqrt{2}} & \frac{\Xi_{c}^{* 0}}{\sqrt{2}} & \Omega_{c}^{* 0}
\end{array}\right]^{\mu}
$$

The leading order chiral Lagrangians for the charmed baryons and light pseudoscalar mesons in the superfield notation read [35-38]

$\mathcal{L}_{\mathcal{B} \varphi}=-\operatorname{Tr}\left[\bar{\psi}^{\mu} i v \cdot D \psi_{\mu}\right]+i g_{a} \epsilon_{\mu \nu \rho \sigma} \operatorname{Tr}\left[\bar{\psi}^{\mu} u^{\rho} v^{\sigma} \psi^{\nu}\right]+i \frac{\delta_{a}}{2} \operatorname{Tr}\left[\bar{\psi}^{\mu} \sigma_{\mu \nu} \psi^{\nu}\right]+\frac{1}{2} \operatorname{Tr}\left[\overline{\mathcal{B}}_{\overline{3}}(i v \cdot D) \mathcal{B}_{\overline{3}}\right]+g_{b} \operatorname{Tr}\left[\bar{\psi}^{\mu} u_{\mu} \mathcal{B}_{\overline{3}}+\right.$ H.c. $]$,

where $\operatorname{Tr}[\cdots]$ denotes the trace in flavor space. The covariant derivative $D_{\mu} \psi=\partial_{\mu} \psi+\Gamma_{\mu} \psi+\psi \Gamma_{\mu}^{T} . \quad g_{a} \simeq$ -1.47 and $g_{b} \simeq 1.04$ are the axial couplings [39-41]. $\delta_{a}=$ $m_{\Xi_{c}^{*}}-m_{\Xi_{c}^{\prime}}$ is the mass splitting between $\Xi_{c}^{*}$ and $\Xi_{c}^{\prime}$ in this calculation. $v^{\mu}=(1, \mathbf{0})$ represents the four-velocity of a slowly moving heavy baryon. The superfields $\psi^{\mu}$ and $\bar{\psi}^{\mu}$ are defined as

$$
\begin{aligned}
\psi^{\mu} & =\mathcal{B}_{6}^{* \mu}-\frac{1}{\sqrt{3}}\left(\gamma^{\mu}+v^{\mu}\right) \gamma^{5} \mathcal{B}_{6}, \\
\bar{\psi}^{\mu} & =\overline{\mathcal{B}}_{6}^{* \mu}+\frac{1}{\sqrt{3}} \overline{\mathcal{B}}_{6} \gamma^{5}\left(\gamma^{\mu}+v^{\mu}\right) .
\end{aligned}
$$

In addition, the chiral connection $\Gamma^{\mu}$ and axial-vector current $u^{\mu}$ read respectively

$$
\Gamma_{\mu} \equiv \frac{1}{2}\left[\xi^{\dagger}, \partial_{\mu} \xi\right], \quad u_{\mu} \equiv \frac{i}{2}\left\{\xi^{\dagger}, \partial_{\mu} \xi\right\}
$$

where

$$
\begin{aligned}
& \xi^{2}=U=\exp \left(\frac{i \varphi}{f_{\pi}}\right), \\
& \varphi=\left[\begin{array}{ccc}
\pi^{0}+\frac{\eta}{\sqrt{3}} & \sqrt{2} \pi^{+} & \sqrt{2} K^{+} \\
\sqrt{2} \pi^{-} & -\pi^{0}+\frac{\eta}{\sqrt{3}} & \sqrt{2} K^{0} \\
\sqrt{2} K^{0} & \sqrt{2} \bar{K}^{0} & -\frac{2 \eta}{\sqrt{3}}
\end{array}\right],
\end{aligned}
$$

and $f_{\pi}=92.4 \mathrm{MeV}$ is the pion decay constant. Expanding Eq. (2) one can get the coupling terms among $\mathcal{B}_{\overline{3}}, \mathcal{B}_{6}$ and 
$\mathcal{B}_{6}^{* \mu}$. The detailed forms and the corresponding axial couplings can be found in Refs. [11,39-41].

The leading order Lagrangians that delineate the interactions between the anticharmed mesons and light Goldstones read $[42,43]$

$$
\mathcal{L}_{\tilde{\mathcal{H}} \varphi}=-i\langle\overline{\tilde{\mathcal{H}}} v \cdot \mathcal{D} \tilde{\mathcal{H}}\rangle-\frac{1}{8} \delta_{b}\left\langle\overline{\tilde{\mathcal{H}}} \sigma^{\mu \nu} \tilde{\mathcal{H}} \sigma_{\mu \nu}\right\rangle+g\left\langle\overline{\tilde{\mathcal{H}}} d l \gamma_{5} \tilde{\mathcal{H}}\right\rangle,
$$

where $\langle\cdots\rangle$ represents the trace in spinor space. The covariant derivative $\mathcal{D}_{\mu}=\partial_{\mu}+\Gamma_{\mu} . \delta_{b}$ is defined as $\delta_{b}=m_{\bar{D}^{*}}-m_{\bar{D}} \cdot g \simeq-0.59$ stands for the axial coupling, which can be extracted from the partial decay width of $D^{*+} \rightarrow D^{0} \pi^{+}$[1]. The $\tilde{\mathcal{H}}$ is the super-field for the anticharmed mesons, which reads

$$
\tilde{\mathcal{H}}=\left[\tilde{P}_{\mu}^{*} \gamma^{\mu}+i \tilde{P} \gamma_{5}\right] \frac{1-\not p}{2}, \quad \overline{\tilde{\mathcal{H}}}=\frac{1-\not \varphi}{2}\left[\tilde{P}_{\mu}^{* \dagger} \gamma^{\mu}+i \tilde{P}^{\dagger} \gamma_{5}\right]
$$

with $\quad \tilde{P}=\left(\bar{D}^{0}, D^{-}, D_{s}^{-}\right)^{T} \quad$ and $\quad \tilde{P}^{*}=\left(\bar{D}^{* 0}, D^{*-}, D_{s}^{*-}\right)^{T}$, respectively.

The contact Lagrangians that describe the short distance interactions between the charmed baryon sextets and anticharmed mesons have been constructed in Refs. $[10,11]$ with the super-field representations, which read

$$
\begin{aligned}
\mathcal{L}_{\tilde{\mathcal{H}} \mathcal{B}_{6}^{(*)}}= & D_{a}\langle\overline{\tilde{\mathcal{H}}} \tilde{\mathcal{H}}\rangle \operatorname{Tr}\left[\bar{\psi}^{\mu} \psi_{\mu}\right] \\
& +i D_{b} \epsilon_{\sigma \mu \nu \rho} v^{\sigma}\left\langle\overline{\tilde{\mathcal{H}}} \gamma^{\rho} \gamma_{5} \tilde{\mathcal{H}}\right\rangle \operatorname{Tr}\left[\bar{\psi}^{\mu} \psi^{\nu}\right] \\
& +E_{a}\left\langle\overline{\tilde{\mathcal{H}}} \lambda^{i} \tilde{\mathcal{H}}\right\rangle \operatorname{Tr}\left[\bar{\psi}^{\mu} \lambda_{i} \psi_{\mu}\right] \\
& +i E_{b} \epsilon_{\sigma \mu \nu \rho} v^{\sigma}\left\langle\tilde{\tilde{\mathcal{H}}} \gamma^{\rho} \gamma_{5} \lambda^{i} \tilde{\mathcal{H}}\right\rangle \operatorname{Tr}\left[\bar{\psi}^{\mu} \lambda_{i} \psi^{\nu}\right]
\end{aligned}
$$

where $D_{a}, D_{b}, E_{a}$, and $E_{b}$ are the LECs. They can be determined by fitting the $P_{c}$ spectra. $\lambda_{i}$ denotes the GellMann matrices.

Besides, we also need the Lagrangians to depict the contact interactions of the charmed baryon antitriplet and anticharmed mesons. They can be analogously constructed as follows,

$$
\begin{aligned}
\mathcal{L}_{\tilde{\mathcal{H}} \mathcal{B}_{\overline{3}}}= & \tilde{D}_{a}\langle\overline{\tilde{\mathcal{H}}} \tilde{\mathcal{H}}\rangle \operatorname{Tr}\left[\overline{\mathcal{B}}_{\overline{3}} \mathcal{B}_{\overline{3}}\right]+\tilde{D}_{b}\left\langle\overline{\tilde{\mathcal{H}}} \gamma^{\rho} \gamma_{5} \tilde{\mathcal{H}}\right\rangle \operatorname{Tr}\left[\overline{\mathcal{B}}_{\overline{3}} \gamma_{\rho} \gamma_{5} \mathcal{B}_{\overline{3}}\right] \\
& +\tilde{E}_{a}\left\langle\overline{\tilde{\mathcal{H}}} \lambda^{i} \tilde{\mathcal{H}}\right\rangle \operatorname{Tr}\left[\overline{\mathcal{B}}_{\overline{3}} \lambda_{i} \mathcal{B}_{\overline{3}}\right] \\
& +\tilde{E}_{b}\left\langle\overline{\tilde{\mathcal{H}}} \gamma^{\rho} \gamma_{5} \lambda^{i} \tilde{\mathcal{H}}\right\rangle \operatorname{Tr}\left[\overline{\mathcal{B}}_{\overline{3}} \gamma_{\rho} \gamma_{5} \lambda_{i} \mathcal{B}_{\overline{3}}\right]
\end{aligned}
$$

where $\tilde{D}_{a}, \tilde{D}_{b}, \tilde{E}_{a}$, and $\tilde{E}_{b}$ are another sets of the LECs. These LECs are different from the ones in Eq. (7), since the $\mathcal{B}_{\overline{3}}$ and $\mathcal{B}_{6}^{(*)}$ are not the partner states under heavy quark spin symmetry. But we can establish the corresponding relationship with the $D_{a}, D_{b}, E_{a}$, and $E_{b}$ with the help of quark model. We show this operation in the Appendix A.

\section{EXPRESSIONS OF THE EFFECTIVE POTENTIALS}

There exists a simple relation between the effective potential and scattering amplitude in momentum space under the Breit approximation,

$$
\mathcal{V}(\boldsymbol{q})=-\frac{\mathcal{M}(\boldsymbol{q})}{\sqrt{2 \Pi_{i} m_{i} 2 \Pi_{f} m_{f}}},
$$

where $m_{i}$ and $m_{f}$ are the masses of the initial and final states. $\boldsymbol{q}$ denotes the transferred momentum between two scattering particles. Then the effective potential in coordinate space can be easily obtained by Fourier transformation, which yields

$$
\mathcal{V}(r)=\int \frac{d^{3} \boldsymbol{q}}{(2 \pi)^{3}} e^{i \boldsymbol{q} \cdot \boldsymbol{r}} \mathcal{V}(\boldsymbol{q}) \mathcal{F}(\boldsymbol{q}),
$$

where a Gauss regulator $\mathcal{F}(\boldsymbol{q})=\exp \left(-\boldsymbol{q}^{2 n} / \Lambda^{2 n}\right)$ is introduced to suppress the high momentum contribution $[44,45] . n=2$ is used in this work [46,47]. Considering the $\rho$ meson mass $m_{\rho}$ is treated as the typical hard scale in chiral effective theory, thus the cutoff $\Lambda$ should be smaller than $m_{\rho}$ (A detailed discussion on the range of $\Lambda$ can be found in Ref. [11]). The $\Lambda$ is chosen to be around $0.5 \mathrm{GeV}$ to perform fittings and give predictions $[10,11,32,46]$.

The topological diagrams are shown in Fig. 1. There are three types of Feynman diagrams in our calculations, i.e., the leading order contact interaction, one-pion-exchange diagram, and the next-to-leading order two-pion-exchange diagrams. The Feynman diagrams for the $\Xi_{c} \bar{D}^{(*)}\left(\Xi_{c}^{\prime} \bar{D}^{(*)}\right)$ and $\Xi_{c}^{*} \bar{D}^{(*)}$ systems are totally the same as the $\Sigma_{c} \bar{D}^{(*)}$ and $\Sigma_{c}^{*} \bar{D}^{(*)}$, respectively. In other words, one can build the following correspondence,

$\Xi_{c}^{\prime} \bar{D}^{(*)}\left[\Xi_{c} \bar{D}^{(*)}\right] \Leftrightarrow \Sigma_{c} \bar{D}^{(*)}, \quad \Xi_{c}^{*} \bar{D}^{(*)} \Leftrightarrow \Sigma_{c}^{*} \bar{D}^{(*)}$.

So we do not explicitly show the detailed graphs for each systems at each order. One can find them in Figs. 2-7 of Ref. [11].

In the following, we write down the leading order contact potential of each system, which can be easily obtained by expanding the Lagrangians in Eqs. (7) and (8), respectively.

$$
\begin{gathered}
\mathcal{V}_{\Xi_{c}^{\prime} \bar{D}}^{X_{i, j}}=-D_{a}-2 \mathcal{G} E_{a}, \\
\mathcal{V}_{\Xi_{c}^{\prime} \bar{D}^{*}}^{X_{i, j}}=-D_{a}-2 \mathcal{G} E_{a}-\frac{2}{3}\left(D_{b}+2 \mathcal{G} E_{b}\right) \boldsymbol{\sigma} \cdot \mathbf{T},
\end{gathered}
$$




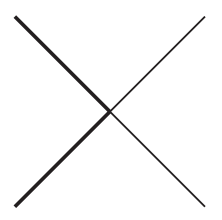

$\left(X_{i, j}\right)$

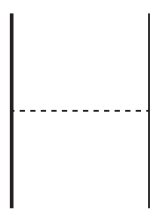

$\left(H_{i, j}\right)$

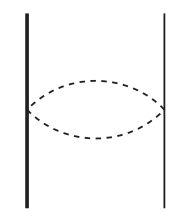

$\left(F_{i, j}\right)$

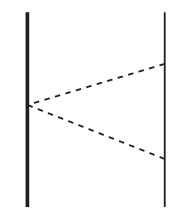

$\left(T_{i, j}\right)$

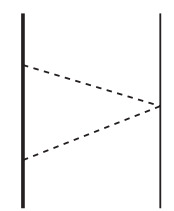

$\left(T_{i . j}\right)$

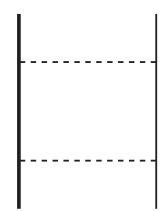

$\left(B_{i, j}\right)$

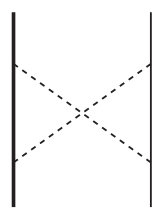

$\left(R_{i, j}\right)$

FIG. 1. Topological Feynman diagrams for the $\Xi_{c} \bar{D}^{(*)}, \Xi_{c}^{\prime} \bar{D}^{(*)}$ and $\Xi_{c}^{*} \bar{D}^{(*)}$ systems. We use the thick line, thin line, and dashed line to denote the charmed baryons, anticharmed mesons and pion, respectively. Graphs $\left(X_{i . j}\right)$ and $\left(H_{i . j}\right)$ stand for the leading order contact interaction and one-pion-exchange diagram, respectively [Diagram $\left(H_{i . j}\right)$ vanishes for the system containing one $\bar{D}$ meson]. $\left(F_{i . j}\right),\left(T_{i . j}\right)$, $\left(B_{i . j}\right)$, and $\left(R_{i . j}\right)$ represent topological structures of two-pion-exchange diagrams, respectively.

$$
\begin{gathered}
\mathcal{V}_{\Xi_{c}^{*} \bar{D}}^{X_{i, j}}=-D_{a}-2 \mathcal{G} E_{a} \\
\mathcal{V}_{\Xi_{c}^{*} \bar{D}^{*}}^{X_{i, j}}=-D_{a}-2 \mathcal{G} E_{a}-\left(D_{b}+2 \mathcal{G} E_{b}\right) \boldsymbol{\sigma}_{r s} \cdot \mathbf{T} \\
\mathcal{V}_{\Xi_{c} \bar{D}}^{X_{i, j}}=2 \tilde{D}_{a}+4 \mathcal{G} \tilde{E}_{a} \\
\mathcal{V}_{\Xi_{c} \tilde{D}^{*}}^{X_{i . j}}=2 \tilde{D}_{a}+4 \mathcal{G} \tilde{E}_{a}+\left(2 \tilde{D}_{b}+4 \mathcal{G} \tilde{E}_{b}\right) \boldsymbol{\sigma} \cdot \mathbf{T},
\end{gathered}
$$

where $\mathcal{G}=\mathbf{I}_{1} \cdot \mathbf{I}_{2}-1 / 12\left[\mathbf{I}_{1}\right.$ and $\mathbf{I}_{2}$ are the isospin operators of $\Xi_{c}^{\prime}\left(\Xi_{c}^{*}, \Xi_{c}\right)$ and $\bar{D}^{(*)}$, respectively]. The operators $\boldsymbol{\sigma}, \boldsymbol{\sigma}_{r s}$ and $\mathbf{T}$ are related to the spin operators of the spin- $\frac{1}{2}$ baryon, spin- $\frac{3}{2}$ baryon, and spin- 1 meson as $\frac{1}{2} \boldsymbol{\sigma}, \frac{3}{2} \boldsymbol{\sigma}_{r s}$, and $-\mathbf{T}$, respectively (see Ref. [11] for details).

The expressions of the one-pion-exchange diagrams for $\Xi_{c}^{\prime} \bar{D}^{*}$ and $\Xi_{c}^{*} \bar{D}^{*}$ are the same as the $\Sigma_{c} \bar{D}^{*}$ and $\Sigma_{c}^{*} \bar{D}^{*}$ in Ref. [11] up to different matrix elements of $\mathbf{I}_{1} \cdot \mathbf{I}_{2}$ operator. For $\Xi_{c} \bar{D}^{*}$, the coupling between $\Xi_{c}$ and $\pi$ vanishes since the pion does not couple to the scalar isoscalar light diquark within $\Xi_{c}$ because of the parity and angular momentum conservation. Thus the one-pionexchange does not contribute to the effective potential of the $\Xi_{c} \bar{D}^{*}$ system.

For the two-pion-exchange diagrams, the graph $\left(F_{i . j}\right)$ is governed by the chiral connection term, thus all the systems share one single expression, i.e.,

$$
\mathcal{V}_{\mathrm{Sys}}^{F_{i, j}}=\left(\mathbf{I}_{1} \cdot \mathbf{I}_{2}\right) \frac{1}{f_{\pi}^{4}} J_{22}^{F}\left(m_{\pi}, q\right)
$$

For graphs $\left(T_{i . j}\right),\left(B_{i . j}\right)$, and $\left(R_{i . j}\right)$, their analytical expressions generally have the following structures,

$\mathcal{V}_{\mathrm{Sys}}^{T_{i, j}}=\left(\mathbf{I}_{1} \cdot \mathbf{I}_{2}\right) \frac{\mathcal{C}_{\mathrm{Sys}}^{T_{i, j}}}{f_{\pi}^{4}}\left[\mathcal{C}_{1}^{T_{i, j}} J_{34}^{T}-\boldsymbol{q}^{2} \mathcal{C}_{2}^{T_{i, j}}\left(J_{24}^{T}+J_{33}^{T}\right)\right]\left(m_{\pi}, \omega, q\right)$,

$$
\begin{aligned}
\mathcal{V}_{\text {Sys }}^{B_{i, j}}= & \left(\frac{1}{8}-\frac{1}{3} \mathbf{I}_{1} \cdot \mathbf{I}_{2}\right) \frac{\mathcal{C}_{\text {Sys }}^{B_{i, j}}}{f_{\pi}^{4}}\left[\mathcal{C}_{1}^{B_{i, j}} J_{41}^{B}\right. \\
& -\boldsymbol{q}^{2} \mathcal{C}_{2}^{B_{i, j}}\left(J_{31}^{B}+J_{42}^{B}\right)-\boldsymbol{q}^{2} \mathcal{C}_{3}^{B_{i, j}} J_{21}^{B} \\
& \left.+\boldsymbol{q}^{4} \mathcal{C}_{4}^{B_{i, j}}\left(J_{22}^{B}+2 J_{32}^{B}+J_{43}^{B}\right)\right]\left(m_{\pi}, \omega, \delta, q\right), \\
\mathcal{V}_{\text {Sys }}^{R_{i, j}}= & \left(\frac{1}{8}+\frac{1}{3} \mathbf{I}_{1} \cdot \mathbf{I}_{2}\right) \frac{\mathcal{C}_{\text {Sys }}^{R_{i, j}}}{f_{\pi}^{4}}\left[\mathcal{C}_{1}^{R_{i, j}} J_{41}^{R}\right. \\
& -\boldsymbol{q}^{2} \mathcal{C}_{2}^{R_{i, j}}\left(J_{31}^{R}+J_{42}^{R}\right)-\boldsymbol{q}^{2} \mathcal{C}_{3}^{R_{i, j}} J_{21}^{R} \\
& \left.+\boldsymbol{q}^{4} \mathcal{C}_{4}^{R_{i, j}}\left(J_{22}^{R}+2 J_{32}^{R}+J_{43}^{R}\right)\right]\left(m_{\pi}, \omega, \delta, q\right),
\end{aligned}
$$

where the subscript "Sys." denotes the corresponding system, such as $\Xi_{c}^{\prime} \bar{D}^{(*)}$ and so on. The superscript " $T_{i . j}$ ", " $B_{i . j}$ " and " $R_{i . j}$ " represent the labels of the Feynman diagrams. Various $J$ functions, such as $J_{x}^{T}, J_{x}^{B}$ and $J_{x}^{R}$ are the scalar loop functions, which are defined and given in Refs. [10,11,26]. The coefficients $\mathcal{C}_{i}^{T_{i . j}}(i=1,2)$ and $\mathcal{C}_{i}^{B_{i, j}}\left(\mathcal{C}_{i}^{R_{i . j}}\right)(i=1, \ldots, 4)$ in the square brackets are the same as the expressions for $\Sigma_{c}^{(*)} \bar{D}^{(*)}$ [11]. According to the correspondence in Eq. (11), one can easily get them by matching with the results in Ref. [11]. The coefficients $\mathcal{C}_{\text {Sys }}^{T_{i, j}}$ and $\mathcal{C}_{\text {Sys }}^{B_{i, j}}\left(\mathcal{C}_{\text {Sys }}^{R_{i, j}}\right)$ for each system are given in Tables I and II.

TABLE I. The coefficients $\mathcal{C}_{\mathrm{Sys}}^{T_{i, j}}$ and $\mathcal{C}_{\mathrm{Sys}}^{B_{i, j}}$ for the systems $\Xi_{c}^{\prime} \bar{D}$, $\Xi_{c} \bar{D}$, and $\Xi_{c}^{*} \bar{D}$, respectively. $i=1$ for $\Xi_{c}^{\prime} \bar{D}\left(\Xi_{c} \bar{D}\right)$ and $i=3$ for $\Xi_{c}^{*} \bar{D}(i$ is the value in the subscript of the diagram label), where the corresponding diagrams can be found in Figs. 3 and 6 of Ref. [11], respectively. The unlisted coefficients $\mathcal{C}_{\text {Sys }}^{R_{i, j}}$ can be obtained with $\mathcal{C}_{\text {Sys }}^{R_{i, j}}=\mathcal{C}_{\text {Sys }}^{B_{i, j}}$. Some expressions for $\mathcal{C}_{\text {Sys }}^{\text {label are }}$ denoted as $X \mid Y$, where $X$ and $Y$ represent the intermediate baryon in the loop among and beyond the heavy quark spin multiplet, respectively.

\begin{tabular}{cccccc}
\hline \hline $\mathcal{C}_{\text {Sys }}^{\text {label }}$ & $T_{i .1}$ & $T_{i .2}$ & $T_{i .3}$ & $B_{i .1}$ & $B_{i .2}$ \\
\hline$\Xi_{c}^{\prime} \bar{D}$ & $g^{2}$ & $\frac{1}{4} g_{3}^{2}$ & $\frac{1}{4} g_{1}^{2} \mid \frac{1}{2} g_{2}^{2}$ & $\frac{3}{8} g^{2} g_{1}^{2} \mid \frac{3}{4} g^{2} g_{2}^{2}$ & $\frac{3}{8} g^{2} g_{3}^{2}$ \\
$\Xi_{c} \bar{D}$ & $g^{2}$ & $\frac{1}{2} g_{4}^{2}$ & $g_{6}^{2} \mid \frac{1}{2} g_{2}^{2}$ & $\frac{3}{2} g^{2} g_{6}^{2} \mid \frac{3}{4} g^{2} g_{2}^{2}$ & $\frac{3}{4} g^{2} g_{4}^{2}$ \\
$\Xi_{c}^{*} \bar{D}$ & $g^{2}$ & $\frac{1}{4} g_{5}^{2}$ & $\frac{1}{4} g_{3}^{2} \mid \frac{1}{2} g_{4}^{2}$ & $\frac{3}{8} g^{2} g_{5}^{2}$ & $\frac{3}{8} g^{2} g_{3}^{2} \mid \frac{3}{4} g^{2} g_{4}^{2}$ \\
\hline \hline
\end{tabular}


TABLE II. The coefficients $\mathcal{C}_{\text {Sys }}^{T_{i . j}}$ and $\mathcal{C}_{\text {Sys }}^{B_{i, j}}$ for the systems $\Xi_{c}^{\prime} \bar{D}^{*}, \Xi_{c} \bar{D}^{*}$, and $\Xi_{c}^{*} \bar{D}^{*}$, respectively. $i=2$ for $\Xi_{c}^{\prime} \bar{D}^{*}\left(\Xi_{c} \bar{D}^{*}\right)$ and $i=4$ for $\Xi_{c}^{*} \bar{D}^{*}$ ( $i$ is the value in the subscript of the diagram label), where the corresponding diagrams can be found in figures 5 and 7 of Ref. [11], respectively. The unlisted coefficients $\mathcal{C}_{\text {Sys }}^{R_{i, j}}$ can be obtained with $\mathcal{C}_{\text {Sys }}^{R_{i, j}}=\mathcal{C}_{\text {Sys }}^{B_{i, j}}$. Some expressions for $\mathcal{C}_{\text {Sys }}^{\text {label }}$ are denoted as $X \mid Y$, the implication is the same as that in Table I.

\begin{tabular}{lcccccccc}
\hline \hline $\mathcal{C}_{\text {Sys }}^{\text {label }}$ & $T_{i .1}$ & $T_{i .2}$ & $T_{i .3}$ & $T_{i .4}$ & $B_{i .1}$ & $B_{i .2}$ & $B_{i .3}$ & $B_{i .4}$ \\
\hline$\Xi_{c}^{\prime} \bar{D}^{*}$ & $g^{2}$ & $g^{2}$ & $\frac{1}{4} g_{1}^{2} \mid \frac{1}{2} g_{2}^{2}$ & $\frac{1}{4} g_{3}^{2}$ & $\frac{3}{8} g^{2} g_{1}^{2} \mid \frac{3}{4} g^{2} g_{2}^{2}$ & $\frac{3}{8} g^{2} g_{1}^{2} \mid \frac{3}{4} g^{2} g_{2}^{2}$ & $\frac{3}{8} g^{2} g_{3}^{2}$ & $\frac{3}{8} g^{2} g_{3}^{2}$ \\
$\Xi_{c} \bar{D}^{*}$ & $g^{2}$ & $g^{2}$ & $g_{6}^{2} \mid \frac{1}{2} g_{2}^{2}$ & $\frac{1}{2} g_{4}^{2}$ & $\frac{3}{2} g^{2} g_{6}^{2} \mid \frac{3}{4} g^{2} g_{2}^{2}$ & $\frac{3}{2} g^{2} g_{6}^{2} \mid \frac{3}{4} g^{2} g_{2}^{2}$ & $\frac{3}{4} g^{2} g_{4}^{2}$ & $\frac{3}{4} g^{2} g_{4}^{2}$ \\
$\Xi_{c}^{*} \bar{D}^{*}$ & $g^{2}$ & $g^{2}$ & $\frac{1}{4} g_{5}^{2}$ & $\frac{1}{4} g_{3}^{2} \mid \frac{1}{2} g_{4}^{2}$ & $\frac{3}{8} g^{2} g_{5}^{2}$ & $\frac{3}{8} g^{2} g_{5}^{2}$ & $\frac{3}{32} g^{2} g_{3}^{2} \mid \frac{3}{16} g^{2} g_{4}^{2}$ & $\frac{3}{32} g^{2} g_{3}^{2} \mid \frac{3}{16} g^{2} g_{4}^{2}$ \\
\hline \hline
\end{tabular}

\section{POSSIBLE MOLECULAR PENTAQUARKS IN $\boldsymbol{\Xi}_{c} \overline{\boldsymbol{D}}^{(*)}, \boldsymbol{\Xi}_{c}^{\prime} \overline{\boldsymbol{D}}^{(*)}$, AND $\boldsymbol{\Xi}_{c}^{*} \overline{\boldsymbol{D}}^{(*)}$ SYSTEMS}

In order to get the numerical results, we have to determine the eight LECs in Eqs. (7) and (8). As in Ref. [48], we also propose a SU(3) quark model to estimate the LECs. One can find the detailed derivations in Appendix A.

The effective potentials of some representative $I=0$ channels are given in Fig. 2. We notice that the leading order contact interaction supplies a strong attractive potential for all the considered channels. This situation is the same as those of the $\Sigma_{c}^{(*)} \bar{D}^{(*)}$ systems [11]. For the $\Xi_{c}^{\prime} \bar{D}$ and $\Xi_{c} \bar{D}^{*}$ systems, one can find the two-pion-exchange contributions are also significant because of the accidental degeneration between $\Xi_{c}^{\prime} \bar{D}$ and $\Xi_{c} \bar{D}^{*}$ in the loops. However, the behavior of the two-pion-exchange potentials for $\Xi_{c}^{\prime} \bar{D}$ and $\Sigma_{c} \bar{D}$ are totally different due to the opposite sign of the mass differences. For example, the mass difference between $\Sigma_{c} \bar{D}$ and $\Lambda_{c} \bar{D}^{*}$ is about $28 \mathrm{MeV}$, while that for $\Xi_{c}^{\prime} \bar{D}$ and $\Xi_{c} \bar{D}^{*}$ is about $-32 \mathrm{MeV}$. By solving the
Schrödinger equation, we find the bound states in the $\Xi_{c}^{\prime} \bar{D}^{(*)}$ and $\Xi_{c}^{*} \bar{D}^{(*)}$ systems, likewise. The predicted binding energies and masses are given in Table III.

Theoretically, the existence of the bound states in the $\Xi_{c}^{\prime} \bar{D}^{(*)}$ and $\Xi_{c}^{*} \bar{D}^{(*)}$ systems is not a surprise, because $\Xi_{c}^{\prime}$ and $\Xi_{c}^{*}$ belong to the same flavor multiplets with the $\Sigma_{c}$ and $\Sigma_{c}^{*}$, respectively in the $\mathrm{SU}(3)$ flavor symmetry [49]. Nevertheless, things become interesting when we go to the $\Xi_{c} \bar{D}^{(*)}$ systems. On the one hand, no bound states are experimentally observed near the $\Lambda_{c} \bar{D}^{(*)}$ thresholds up to now [9]. On the other hand, some theoretical calculations also do not support the existence of bound states in $\Lambda_{c} \bar{D}^{(*)}$ systems $[50,51]$. As the $\mathrm{SU}(3)$ partner of $\Lambda_{c}$, the interactions of the $\Xi_{c} \bar{D}^{(*)}$ systems show totally different behavior at the short range [e.g., see Figs. $2(e)$ and $2(f)]$. The attractive contact interaction is strong enough to form bound states.

For the $\Lambda_{c} \bar{D}^{(*)}$ and $\Xi_{c} \bar{D}^{(*)}$ systems, the long-range onepion-exchange vanishes, thus only the contact term and twopion-exchange contribute to their potentials. The isospin $I$
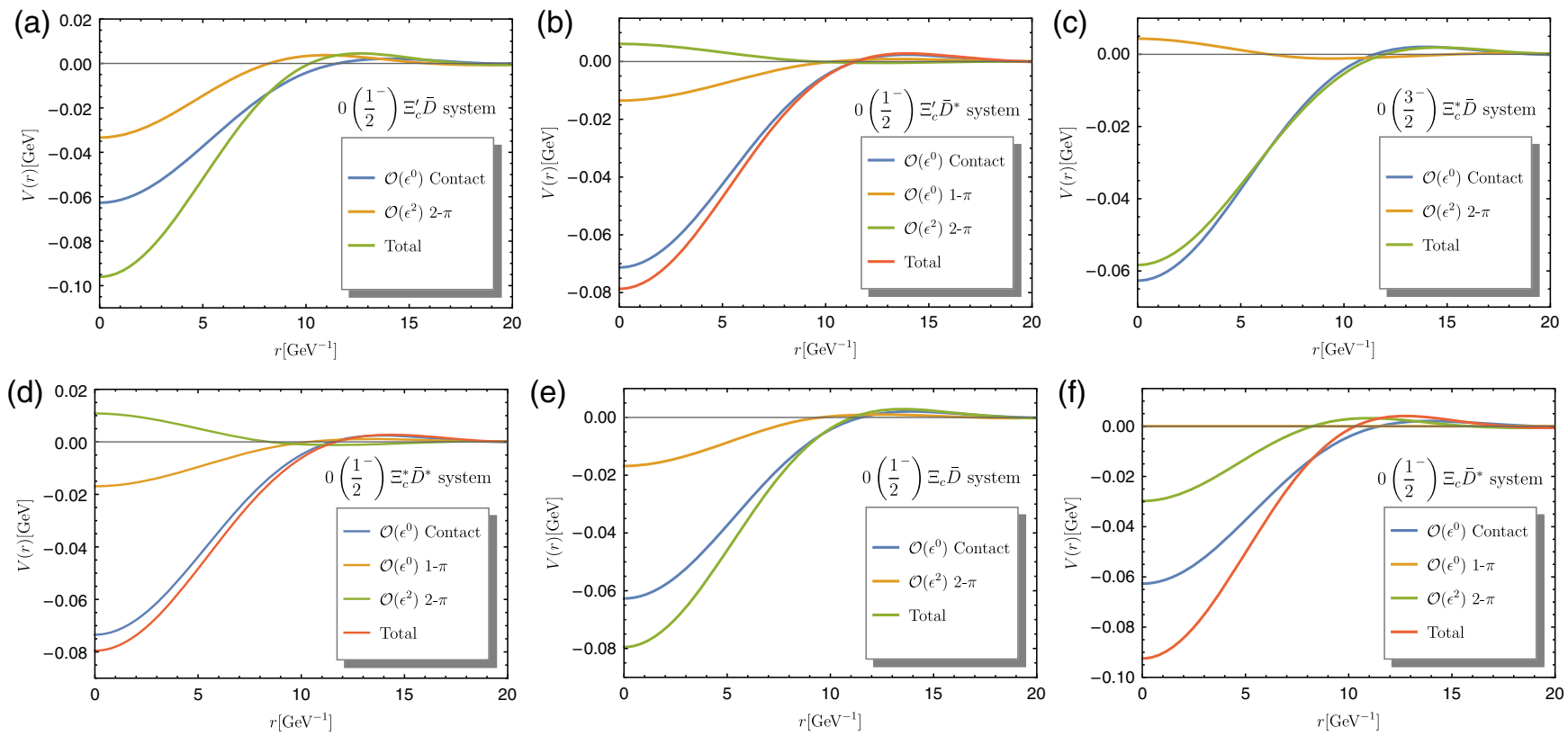

FIG. 2. The effective potentials of the $\Xi_{c}^{\prime} \bar{D}^{(*)}, \Xi_{c}^{*} \bar{D}^{(*)}$, and $\Xi_{c} \bar{D}^{(*)}$ systems. Their $I\left(J^{P}\right)$ are marked in each subfigure. The potentials are obtained with the cutoff parameter $\Lambda=0.4 \mathrm{GeV}$. For the effective potentials of the unlisted spin multiplets, their total potentials have the similar behaviors with the lowest spin states. 
TABLE III. The predicted binding energies $\Delta E$ and masses $M$ for the $\left[\Xi_{c}^{\prime} \bar{D}^{(*)}\right]_{J},\left[\Xi_{c}^{*} \bar{D}^{(*)}\right]_{J}$, and $\left[\Xi_{c} \bar{D}^{(*)}\right]_{J}$ systems in $I=0$ channel, where the subscript " $J$ " denotes the total spin of the system. We correspondingly use the thresholds of $\Xi_{c}^{\prime+} \bar{D}^{(*) 0}, \Xi_{c}^{*+} \bar{D}^{(*) 0}$, and $\Xi_{c}^{+} \bar{D}^{(*) 0}$ as the benchmarks to calculate the values in this table (in units of MeV). The state that denoted by " $\sharp$ " means which may be nonexistent at the upper limit.

\begin{tabular}{|c|c|c|c|c|c|c|c|c|c|c|}
\hline System & {$\left[\Xi_{c}^{\prime} \bar{D}\right]_{\frac{1}{2}}$} & {$\left[\Xi_{c}^{\prime} \bar{D}^{*}\right]_{\frac{1}{2}}$} & {$\left[\Xi_{c}^{\prime} \bar{D}^{*}\right]_{\frac{3}{2}}$} & {$\left[\Xi_{c}^{*} \bar{D}\right]_{\frac{3}{2}}$} & {$\left[\Xi_{c}^{*} \bar{D}^{*}\right]_{\frac{1}{2}}$} & {$\left[\Xi_{c}^{*} \bar{D}^{*}\right]_{\frac{3}{2}}$} & {$\left[\Xi_{c}^{*} \bar{D}^{*}\right]_{\frac{5}{2}}^{\#}$} & {$\left[\Xi_{c} \bar{D}\right]_{\frac{1}{2}}$} & {$\left[\Xi_{c} \bar{D}^{*}\right]_{\frac{1}{2}}$} & {$\left[\Xi_{c} \bar{D}^{*}\right]_{\frac{3}{2}}$} \\
\hline$\Delta E$ & $-18.5_{-6.8}^{+6.4}$ & $-15.6_{-7.2}^{+6.4}$ & $-2.0_{-3.3}^{+1.8}$ & $-7.5_{-5.3}^{+4.2}$ & $-17.0_{-7.5}^{+6.7}$ & $-8.0_{-5.6}^{+4.5}$ & $-0.7_{-2.2}^{+0.7}$ & $-13.3_{-3.0}^{+2.8}$ & $-17.8_{-3.3}^{+3.2}$ & $-11.8_{-3.0}^{+2.8}$ \\
\hline$M$ & $4423.7_{-6.8}^{+6.4}$ & $4568.7_{-7.2}^{+6.4}$ & $4582.3_{-3.3}^{+1.8}$ & $4502.9_{-5.3}^{+4.2}$ & $4635.4_{-7.5}^{+6.7}$ & $4644.4_{-5.6}^{+4.5}$ & $4651.7_{-2.2}^{+0.7}$ & $4319.4_{-3.0}^{+2.8}$ & $4456.9_{-3.3}^{+3.2}$ & $4463.0_{-3.0}^{+2.8}$ \\
\hline
\end{tabular}

and spin $J^{\ell}\left(J^{\ell}\right.$ denotes the total spin of the light degrees of freedom in $\Lambda_{c}$ ) of the $\Lambda_{c}$ are both zero, so there are no isospin-isospin and spin-spin interactions for the $\Lambda_{c} \bar{D}^{(*)}$ systems. ${ }^{1}$ Ignoring the $\eta$ and $K$ meson contributions in the loops, one can roughly get the two-pion-exchange potential of the $\Lambda_{c} \bar{D}^{(*)}$ from the $\Xi_{c} \bar{D}^{(*)}$ expressions by setting the matrix element $\left\langle\mathbf{I}_{1} \cdot \mathbf{I}_{2}\right\rangle$ to be zero. Although the two-pionexchange potential of the $\Lambda_{c} \bar{D}^{(*)}$ (or say the contribution from couple-channel effect) is attractive, the one from the leading order $\tilde{D}_{a}$ term [e.g., see Eq. (8)] is repulsive. Their contributions almost cancel each other, thus there are no binding solutions for the $\Lambda_{c} \bar{D}^{(*)}$ systems.

The $J^{\ell}$ of $\Xi_{c}$ is also zero, thus there are no spin-spin interactions for the $\Xi_{c} \bar{D}^{(*)}$ systems as well. But $I=\frac{1}{2}$ for $\Xi_{c}$, i.e., the isospin-isospin interaction in the $\Xi_{c} \bar{D}^{(*)}$ systems is the main reason that leads to the different scenarios for the $\Lambda_{c} \bar{D}^{(*)}$ and $\Xi_{c} \bar{D}^{(*)}$ systems. The leading order isospin related $\tilde{E}_{a}$ term provides a very strong attractive potential, as well as the attractive two-pionexchange potential. Their contributions together yield three bound states in the $\Xi_{c} \bar{D}^{(*)}$ systems (see Table III). A recent study based on the local hidden gauge approach also gives a similar conclusion [52].

We also tried to include the one-eta-exchange contribution for the $\Xi_{c}^{\prime} \bar{D}^{*}$ and $\Xi_{c}^{*} \bar{D}^{*}$ systems at the leading order, where we use the experimental values of $f_{\eta}$ and $m_{\eta}$ as inputs [39-41,53]. We notice the one-eta-exchange contribution is marginal, which only introduces about $1 \%$ and even much smaller than $1 \%$ corrections to the $\Xi_{c}^{\prime} \bar{D}^{*}$ and $\Xi_{c}^{*} \bar{D}^{*}$ binding energies, respectively.

Additionally, we also calculate the potentials of these six systems in the $I=1$ channel. However, the total potentials are all repulsive, i.e., no bound states exist for the $\Xi_{c} \bar{D}^{(*)}$, $\Xi_{c}^{\prime} \bar{D}^{(*)}$, and $\Xi_{c}^{*} \bar{D}^{(*)}$ systems in the isovector channels.

These $I=0$ molecular pentaquarks with a strange quark can be reconstructed at the $J / \psi \Lambda$ final states. We hope LHCb collaborations may search for these new $P_{c s}$ s near the $\Xi_{c} \bar{D}^{(*)}$ thresholds. Some discussions on the $\Lambda_{c} \bar{D}_{s}^{(*)}, \Sigma_{c} \bar{D}_{s}^{(*)}$, $\Sigma_{c}^{*} \bar{D}_{s}^{(*)}$, and $\Omega_{c}^{(*)} \bar{D}_{s}^{(*)}$ systems are given in Appendix B.

\footnotetext{
${ }^{1}$ At the leading order of the heavy quark expansion, the spinspin interaction between $\Lambda_{c}$ and $\bar{D}^{*}$ are represented by their light degrees of freedom, i.e., $\boldsymbol{J}_{\Lambda_{c}}^{\ell} \cdot \boldsymbol{J}_{\bar{D}^{*}}^{\ell}$. Its matrix element vanishes for the $\Lambda_{c} \bar{D}^{*}$ system.
}

\section{SUMMARY}

In this work, we have systematically calculated the effective potentials of $\Xi_{c} \bar{D}^{(*)}, \Xi_{c}^{\prime} \bar{D}^{(*)}$, and $\Xi_{c}^{*} \bar{D}^{(*)}$ systems with the chiral effective field theory up to the next-to-leading order. The contact interaction, one-pion-exchange contribution and twopion-exchange diagrams are considered. By fitting the newly observed $P_{c}$ spectra, we relate the LECs to those of the $\Sigma_{c} \bar{D}^{*}$ systems with the quark model (see Appendix A).

As the partners of $\Sigma_{c} \bar{D}^{(*)}$ and $\Sigma_{c}^{*} \bar{D}^{(*)}$, we also find seven bound states in the isoscalar $\left[\Xi_{c}^{\prime} \bar{D}^{(*)}\right]_{J}$ and $\left[\Xi_{c}^{*} \bar{D}^{(*)}\right]_{J}$ systems. The contact terms provide the attractive potentials, which are dominant for these systems. The two-pionexchange interactions are important to the $\Xi_{c}^{\prime} \bar{D}$ and $\Xi_{c} \bar{D}^{*}$ systems due to the accidental degeneration of the intermediate states in the loops.

With the estimated LECs, we also obtain three bound states in the isoscalar $\left[\Xi_{c} \bar{D}^{(*)}\right]_{J}$ systems. This is very different from their partners $\Lambda_{c} \bar{D}^{(*)}$. Our analyses do not support the existence of any molecular pentaquarks in the $\Lambda_{c} \bar{D}^{(*)}$ systems. The difference between $\Lambda_{c} \bar{D}^{(*)}$ and $\left[\Xi_{c} \bar{D}^{(*)}\right]_{J}$ arises from the isospin-isospin interaction, which vanishes for the $\Lambda_{c} \bar{D}^{(*)}$.

We considered the influence of one-eta-exchange interaction. Its contribution only gives rise to $1 \%$ corrections to the magnitude of the binding energies. Thus the tiny effect is neglected in our numerical results. Our calculation indicates that the potentials of the $I=1$ channels are all strongly repulsive and no bound states exist in these $I=1$ channels.

In summary, we obtain ten molecular pentaquarks $P_{c s}$ in the isoscalar $\Xi_{c} \bar{D}^{(*)}, \Xi_{c}^{\prime} \bar{D}^{(*)}$, and $\Xi_{c}^{*} \bar{D}^{(*)}$ systems. Their signals can be reconstructed in the $J / \psi \Lambda$ final states at $\mathrm{LHCb}$ experiment.

\section{ACKNOWLEDGMENTS}

B. Wang is very grateful to R. Chen, X. L. Chen and W.Z. Deng for helpful discussions. This project is supported by the National Natural Science Foundation of China under Grant No. 11975033.

\section{APPENDIX A: ESTIMATING THE LECS WITH QUARK MODEL}

In this part, we estimate the eight LECs in Eqs. (7) and (8) from the viewpoints of the quark model. We assume the 
short-range contact interaction stems from some heavy particle exchanges, which is analogous to the resonance saturation model [54]. However, we do not specify the exchange particles (such as $\rho, \omega, f_{0}$, etc..) as in the oneboson-exchange scheme, because their contributions are partially mimicked by the two-pion-exchange diagrams. Rather than calculating the contact potential at the hadron level, we construct the quark-level Lagrangians to depict the short-range interaction by borrowing some concepts from the quark-hadron duality and quark model. Generally, one can formulate the quark-level Lagrangians as follows,

$$
\mathcal{L}=g_{s} \bar{q} \mathcal{S} q+g_{a} \bar{q} \gamma_{\mu} \gamma^{5} \mathcal{A}^{\mu} q
$$

where $q=(u, d, s), g_{s}$ and $g_{a}$ are two independent coupling constants. The $\mathcal{S}$ and $\mathcal{A}^{\mu}$ are two fictitious fields, which create (annihilate) the scalar and axial-vector spurions respectively. We assume the $\mathcal{S}$ and $\mathcal{A}^{\mu}$ are flavor octets and have the similar matrix form as that in the second term of Eq. (5). They are introduced to produce the central potential and spin-spin interaction between two quarks, respectively.

With the quark-level Lagrangians, the $\Sigma_{c} \bar{D}^{*}$ effective potential can be expressed as

$$
\begin{aligned}
V_{\Sigma_{c} \bar{D}^{*}}^{\mathrm{QL} .}= & \left\langle\Sigma_{c} \bar{D}^{*}|\mathcal{L}| \Sigma_{c} \bar{D}^{*}\right\rangle=-\frac{g_{s}^{2}}{6 m_{\mathcal{S}}^{2}}-\frac{g_{s}^{2}}{m_{\mathcal{S}}^{2}}\left(\mathbf{I}_{1} \cdot \mathbf{I}_{2}\right) \\
& +\frac{g_{a}^{2}}{9 m_{\mathcal{A}}^{2}} \boldsymbol{\sigma} \cdot \mathbf{T}+\frac{2 g_{a}^{2}}{3 m_{\mathcal{A}}^{2}}(\boldsymbol{\sigma} \cdot \mathbf{T})\left(\mathbf{I}_{1} \cdot \mathbf{I}_{2}\right),
\end{aligned}
$$

where the superscript "Q.L." is the abbreviation of quark level. We take the SU(3) flavor symmetry and ignore the mass differences in the $\mathcal{S}$ and $\mathcal{A}^{\mu}$ multiplets, respectively. In addition, the assumption that $q^{2} \ll m_{\mathcal{S}}^{2}\left(m_{\mathcal{A}}^{2}\right)$ is used. Thus if we know the square of the "charge-to-mass ratios" $g_{s}^{2} / m_{\mathcal{S}}^{2}$ and $g_{a}^{2} / m_{\mathcal{A}}^{2}$, we could obtain the potentials of the other systems that contain the light quarks. The contact potential of the $\Sigma_{c} \bar{D}^{*}$ in the $I=\frac{1}{2}$ channel is given as [11]

$$
V_{\Sigma_{c} \bar{D}^{*}}=-\mathbb{D}_{1}-\frac{2}{3} \mathbb{D}_{2}(\boldsymbol{\sigma} \cdot \mathbf{T})
$$

By fitting the $P_{c}$ spectra with the cutoff $\Lambda=0.4 \mathrm{GeV}$, we get $\mathbb{D}_{1}=63.1 \mathrm{GeV}^{-2}, \mathbb{D}_{2}=6.5 \mathrm{GeV}^{-2}$. Defining $C_{1}=$ $g_{s}^{2} / m_{\mathcal{S}}^{2}, \quad C_{2}=g_{a}^{2} / m_{\mathcal{A}}^{2}$, and comparing Eq. (A2) and Eq. (A3), one easily gets

$$
C_{1}=-\frac{6}{5} \mathbb{D}_{1}, \quad C_{2}=\frac{6}{5} \mathbb{D}_{2} .
$$

Following the same procedure, we can also calculate the $\Xi_{c}^{\prime} \bar{D}^{*}$ contact potential with the quark-level Lagrangians, which yield

$$
V_{\Xi_{c}^{\prime} \bar{D}^{*}}^{\mathrm{Q} . \mathrm{L}}=\left[\frac{1}{12}-\left(\mathbf{I}_{1} \cdot \mathbf{I}_{2}\right)\right]\left(C_{1}-\frac{2}{3} C_{2} \boldsymbol{\sigma} \cdot \mathbf{T}\right)
$$

Matching Eq. (13) and Eq. (A5), one can obtain the LECs in Eq. (7) under the SU(3) symmetry, which read

$D_{a}=0, \quad D_{b}=0, \quad E_{a}=\frac{1}{2} C_{1}, \quad E_{b}=-\frac{1}{2} C_{2}$.

Similarly, one can also calculate the contact potential of $\Xi_{c} \bar{D}^{*}$ at the quark level with the Lagrangians in Eq. (A1),

$$
V_{\Xi_{c} \bar{D}^{*}}^{\text {Q.L. }}=\left[\frac{1}{12}-\left(\mathbf{I}_{1} \cdot \mathbf{I}_{2}\right)\right] C_{1}
$$

A match between Eq. (17) and Eq. (A7) gives

$$
\tilde{D}_{a}=0, \quad \tilde{D}_{b}=0, \quad \tilde{E}_{a}=-\frac{1}{4} C_{1}, \quad \tilde{E}_{b}=0,
$$

where $\tilde{D}_{b}=\tilde{E}_{b}=0$ is the consequence of $J^{\ell}=0$ for the light diquark in $\Xi_{c}$, i.e., the spin-spin interaction vanishes for the $\mathcal{B}_{\overline{3}}$ baryons.

Because the exchange particles we considered in Eq. (A1) are only octets, thus the final results show that the LECs $D_{a}, D_{b}$, and $\tilde{D}_{a}$ are all zero. Their values should be contributed by the singlets exchange. We could estimate the $D_{a}, D_{b}$, and $\tilde{D}_{a}$ by replacing the octets in $\mathcal{S}$ and $\mathcal{A}$ with the nonet. An alternative way is to expand the Gell-Mann matrices in Eqs. (7) and (8) with $\lambda_{0}=\sqrt{2 / 3} \operatorname{diag}\{1,1,1\}$. The extra $\lambda_{0}$ terms can be matched to the $D_{a}, D_{b}$, and $\tilde{D}_{a}$ terms. The relations read

$$
D_{a}=\frac{2}{3} E_{a}, \quad D_{b}=\frac{2}{3} E_{b}, \quad \tilde{D}_{a}=\frac{2}{3} \tilde{D}_{a} .
$$

We attempt to include the influences of $D_{a}, D_{b}$, and $\tilde{D}_{a}$ on the numerical results in the $\mathrm{SU}(3)$ case. Considering the masses of the singlets are heavier than those of the octets, we adopt the half values in Eq. (A9) as their limits to give a conservative estimation. Finally, the values of these LECs are given in Table IV.

TABLE IV. The values of the LECs in Eqs. (7) and (8) estimated with quark model (in units of $\mathrm{GeV}^{-2}$ ).

\begin{tabular}{lccc}
\hline \hline$D_{a}$ & $D_{b}$ & $E_{a}$ & $E_{b}$ \\
\hline $0 \pm 12.6$ & $0 \pm 1.3$ & -37.9 & -3.9 \\
$\tilde{D}_{a}$ & $\tilde{D}_{b}$ & $\tilde{E}_{a}$ & $\tilde{E}_{b}$ \\
$0 \pm 3.2$ & 0 & 18.9 & 0 \\
\hline \hline
\end{tabular}




\section{APPENDIX B: SOME DISCUSSIONS ON THE} $\Lambda_{c} \overline{\boldsymbol{D}}_{s}^{(*)}, \boldsymbol{\Sigma}_{c} \overline{\boldsymbol{D}}_{s}^{(*)}, \boldsymbol{\Sigma}_{c}^{*} \overline{\boldsymbol{D}}_{s}^{(*)}$, AND $\boldsymbol{\Omega}_{c}^{(*)} \overline{\boldsymbol{D}}_{s}^{(*)}$ SYSTEMS

In addition to the $\Xi_{c} \bar{D}^{(*)}, \Xi_{c}^{\prime} \bar{D}^{(*)}$, and $\Xi_{c}^{*} \bar{D}^{(*)}$ systems we presented above, the strange hidden charm configuration can also be the $\Lambda_{c} \bar{D}_{s}^{(*)}, \Sigma_{c} \bar{D}_{s}^{(*)}$, and $\Sigma_{c}^{*} \bar{D}_{s}^{(*)}$ systems, even the three strange systems $\Omega_{c}^{(*)} \bar{D}_{s}^{(*)}$. In this appendix, we discuss the possibility of the existence of bound states in these systems. There are ten systems by unfolding the different combinations. We first list their leading order contact potentials in the following,

$$
\begin{gathered}
\mathcal{V}_{\Lambda_{c} \bar{D}_{s}}=2 \tilde{D}_{a}-\frac{4}{3} \tilde{E}_{a} \\
\mathcal{V}_{\Lambda_{c} \bar{D}_{s}^{*}}=2 \tilde{D}_{a}-\frac{4}{3} \tilde{E}_{a}+\left[2 \tilde{D}_{b}-\frac{4}{3} \tilde{E}_{b}\right] \boldsymbol{\sigma} \cdot \mathbf{T}, \\
\mathcal{V}_{\Sigma_{c} \bar{D}_{s}}=-D_{a}+\frac{2}{3} E_{a} \\
\mathcal{V}_{\Sigma_{c} \bar{D}_{s}^{*}}=-D_{a}+\frac{2}{3} E_{a}+\frac{2}{3}\left[-D_{b}+\frac{2}{3} E_{b}\right] \boldsymbol{\sigma} \cdot \mathbf{T}, \\
\mathcal{V}_{\Sigma_{c}^{*} \bar{D}_{s}}=-D_{a}+\frac{2}{3} E_{a} \\
\mathcal{V}_{c}^{*} \bar{D}_{s}^{*}=-D_{a}+\frac{2}{3} E_{a}+\left[-D_{b}+\frac{2}{3} E_{b}\right] \boldsymbol{\sigma}_{r s} \cdot \mathbf{T} \\
\mathcal{V}_{\Omega_{c} \bar{D}_{s}}=-D_{a}-\frac{4}{3} E_{a} \\
\mathcal{V}_{\Omega_{c} \bar{D}_{s}^{*}}=-D_{a}-\frac{4}{3} E_{a}+\frac{2}{3}\left[-D_{b}-\frac{4}{3} E_{b}\right] \boldsymbol{\sigma} \cdot \mathbf{T} \\
\mathcal{V}_{\Omega_{c}^{*} \bar{D}_{s}}=-D_{a}-\frac{4}{3} E_{a} \\
\mathcal{V}_{\Omega_{c}^{*} \bar{D}_{s}^{*}}=-D_{a}-\frac{4}{3} E_{a}+\left[-D_{b}-\frac{4}{3} E_{b}\right] \boldsymbol{\sigma}_{r s} \cdot \mathbf{T} .
\end{gathered}
$$

Yet, there does not exist the one-pion-exchange potential for these systems at the leading order. The exchange particle can only be the $\eta$ meson for the $\Sigma_{c} \bar{D}_{s}^{(*)}, \Sigma_{c}^{*} \bar{D}_{s}^{(*)}$, and $\Omega_{c}^{(*)} \bar{D}_{s}^{(*)}$ systems. In the previous sections, we notice that the influence of one-eta-exchange is rather feeble. So the leading order contribution is dominantly from the contact terms.

The situation becomes intractable to the next-to-leading order. On the one hand, since the exchanged particles in the loops are the kaon and $\eta$ meson, their masses are about four times larger than the pion mass. The large mass of kaon and $\eta$ meson would make the loop diagram contribution being immoderately enhanced, which breaks the convergence of chiral expansion. In other words, the prediction is not stable any more in this case. This is why we did not consider the kaon and $\eta$ meson exchange for the $\Xi_{c} \bar{D}^{(*)}, \Xi_{c}^{\prime} \bar{D}^{(*)}$, and $\Xi_{c}^{*} \bar{D}^{(*)}$ systems in the loop diagrams. On the other hand, we obtained the cutoff $\Lambda \simeq 0.4 \mathrm{GeV}$ by fitting the newly observed $P_{c}$ states. The kaon and $\eta$ meson masses are obviously larger than the cutoff, thus the kaon and $\eta$ meson contributions can be regarded as being integrated out and packaged into the LECs. Therefore, we do not try to explicitly calculate the two-kaon- and two-eta-exchange contributions for these systems. Alternatively, we focus on the contact interaction to conservatively investigate the behavior of their potentials.

Using the estimated LECs in Table IV, the potentials of the $\Lambda_{c} \bar{D}_{s}^{(*)}, \Sigma_{c} \bar{D}_{s}^{(*)}$, and $\Sigma_{c}^{*} \bar{D}_{s}^{(*)}$ systems are attractive, but the attractions are too weak to form the bound states. We also tried to add half value to the LECs, but no binding solutions are found yet. The potentials of the $\Omega_{c}^{(*)} \bar{D}_{s}^{(*)}$ systems are all repulsive, thus there also are no bound states in the $\Omega_{c}^{(*)} \bar{D}_{s}^{(*)}$ systems. The investigation on the $\Xi_{c} \bar{D}^{(*)}, \Xi_{c}^{\prime} \bar{D}^{(*)}$, and $\Xi_{c}^{*} \bar{D}^{(*)}$ systems shows that the strong attractive potentials are provided by the isospin-isospin interactions in the leading contact terms. However, for the $\Lambda_{c} \bar{D}_{s}^{(*)}, \Sigma_{c} \bar{D}_{s}^{(*)}, \Sigma_{c}^{*} \bar{D}_{s}^{(*)}$, and $\Omega_{c}^{(*)} \bar{D}_{s}^{(*)}$ systems, there does not exist the isospin-isospin interactions, thus our study indicates it is hard to form the bound states in these systems.
[1] M. Tanabashi et al. (Particle Data Group), Review of particle physics, Phys. Rev. D 98, 030001 (2018).

[2] S. K. Choi et al. (Belle Collaboration), Observation of a Narrow Charmonium-Like State in Exclusive $B^{ \pm} \rightarrow$ $K^{ \pm} \pi^{+} \pi^{-} J / \psi$ Decays, Phys. Rev. Lett. 91, 262001 (2003).

[3] H. X. Chen, W. Chen, X. Liu, and S. L. Zhu, The hidden-charm pentaquark and tetraquark states, Phys. Rep. 639, 1 (2016).

[4] F. K. Guo, C. Hanhart, U. G. Meißner, Q. Wang, Q. Zhao, and B.S. Zou, Hadronic molecules, Rev. Mod. Phys. 90, 015004 (2018).
[5] Y. R. Liu, H. X. Chen, W. Chen, X. Liu, and S. L. Zhu, Pentaquark and tetraquark states, Prog. Part. Nucl. Phys. 107, 237 (2019).

[6] R. F. Lebed, R. E. Mitchell, and E.S. Swanson, Heavy-quark QCD exotica, Prog. Part. Nucl. Phys. 93, 143 (2017).

[7] A. Esposito, A. Pilloni, and A. D. Polosa, Multiquark resonances, Phys. Rep. 668, 1 (2017).

[8] N. Brambilla, S. Eidelman, C. Hanhart, A. Nefediev, C. P. Shen, C. E. Thomas, A. Vairo, and C. Z. Yuan, The $X Y Z$ 
states: Experimental and theoretical status and perspectives, arXiv:1907.07583.

[9] R. Aaij et al. (LHCb Collaboration), Observation of a Narrow Pentaquark State, $P_{c}(4312)^{+}$, and of Two-Peak Structure of the $P_{c}(4450)^{+}$, Phys. Rev. Lett. 122, 222001 (2019).

[10] L. Meng, B. Wang, G. J. Wang, and S. L. Zhu, The hidden charm pentaquark states and $\Sigma_{c} \bar{D}^{(*)}$ interaction in chiral perturbation theory, Phys. Rev. D 100, 014031 (2019).

[11] B. Wang, L. Meng, and S. L. Zhu, Hidden-charm and hidden-bottom molecular pentaquarks in chiral perturbation theory, J. High Energy Phys. 11 (2019) 108.

[12] M. Z. Liu, Y. W. Pan, F. Z. Peng, M. S. Snchez, L. S. Geng, A. Hosaka, and M. P. Valderrama, Emergence of a Complete Heavy-Quark Spin Symmetry Multiplet: Seven Molecular Pentaquarks in Light of the Latest LHCb Analysis, Phys. Rev. Lett. 122, 242001 (2019).

[13] R. Chen, Z. F. Sun, X. Liu, and S. L. Zhu, Strong LHCb evidence supporting the existence of the hidden-charm molecular pentaquarks, Phys. Rev. D 100, 011502 (2019).

[14] C. W. Xiao, J. Nieves, and E. Oset, Heavy quark spin symmetric molecular states from $\bar{D}^{(*)} \Sigma_{c}^{(*)}$ and other coupled channels in the light of the recent $\mathrm{LHCb}$ pentaquarks, Phys. Rev. D 100, 014021 (2019).

[15] J. He, Study of $P_{c}(4457), P_{c}(4440)$, and $P_{c}(4312)$ in a quasipotential Bethe-Salpeter equation approach, Eur. Phys. J. C 79, 393 (2019).

[16] C. J. Xiao, Y. Huang, Y. B. Dong, L. S. Geng, and D. Y. Chen, Exploring the molecular scenario of $P_{c}(4312)$, $P_{c}(4440)$, and $P_{c}(4457)$, Phys. Rev. D 100, 014022 (2019).

[17] M. B. Voloshin, Some decay properties of hidden-charm pentaquarks as baryon-meson molecules, Phys. Rev. D 100, 034020 (2019).

[18] F. K. Guo, H. J. Jing, U. G. Meißner, and S. Sakai, Isospin breaking decays as a diagnosis of the hadronic molecular structure of the $P_{c}$ (4457), Phys. Rev. D 99, 091501 (2019).

[19] Z. H. Guo and J. A. Oller, Anatomy of the newly observed hidden-charm pentaquark states: $P_{c}(4312), P_{c}(4440)$ and $P_{c}$ (4457), Phys. Lett. B 793, 144 (2019).

[20] T. J. Burns and E. S. Swanson, Molecular interpretation of the $P_{c}(4440)$ and $P_{c}(4457)$ states, Phys. Rev. D 100, 114033 (2019).

[21] G. J. Wang, L. Y. Xiao, R. Chen, X. H. Liu, X. Liu, and S. L. Zhu, Probing hidden-charm decay properties of $P_{c}$ states in a molecular scenario, arXiv:1911.09613.

[22] N. Lee, Z. G. Luo, X. L. Chen, and S. L. Zhu, Possible deuteron-like molecular states composed of heavy baryons, Phys. Rev. D 84, 014031 (2011).

[23] N. Li and S. L. Zhu, Hadronic molecular states composed of heavy flavor baryons, Phys. Rev. D 86, 014020 (2012).

[24] X. Q. Li and X. Liu, A possible global group structure for exotic states, Eur. Phys. J. C 74, 3198 (2014).

[25] H. Xu, B. Wang, Z. W. Liu, and X. Liu, DD* potentials in chiral perturbation theory and possible molecular states, Phys. Rev. D 99, 014027 (2019).

[26] B. Wang, Z. W. Liu, and X. Liu, $\bar{B}^{(*)} \bar{B}^{(*)}$ interactions in chiral effective field theory, Phys. Rev. D 99, 036007 (2019).

[27] J. X. Lu, E. Wang, J. J. Xie, L. S. Geng, and E. Oset, The $\Lambda_{b} \rightarrow J / \psi K^{0} \Lambda$ reaction and a hidden-charm pentaquark state with strangeness, Phys. Rev. D 93, 094009 (2016).
[28] A. Feijoo, V. K. Magas, A. Ramos, and E. Oset, A hiddencharm $S=-1$ pentaquark from the decay of $\Lambda_{b}$ into $J / \psi \eta \Lambda$ states, Eur. Phys. J. C 76, 446 (2016).

[29] H. X. Chen, L. S. Geng, W. H. Liang, E. Oset, E. Wang, and J. J. Xie, Looking for a hidden-charm pentaquark state with strangeness $S=-1$ from $\Xi_{b}^{-}$decay into $J / \psi K^{-} \Lambda$, Phys. Rev. C 93, 065203 (2016).

[30] V. Bernard, N. Kaiser, and U. G. Meißner, Chiral dynamics in nucleons and nuclei, Int. J. Mod. Phys. E 04, 193 (1995).

[31] E. Epelbaum, H. W. Hammer, and U. G. Meißner, Modern theory of nuclear forces, Rev. Mod. Phys. 81, 1773 (2009).

[32] R. Machleidt and D. R. Entem, Chiral effective field theory and nuclear forces, Phys. Rep. 503, 1 (2011).

[33] U. G. Meißner, The long and winding road from chiral effective Lagrangians to nuclear structure, Phys. Scripta 91, 033005 (2016).

[34] H.-W. Hammer, S. König, and U. van Kolck, Nuclear effective field theory: Status and perspectives, arXiv:1906.12122.

[35] P. L. Cho, Heavy hadron chiral perturbation theory, Nucl. Phys. B396, 183 (1993).

[36] H. Y. Cheng, C. Y. Cheung, G. L. Lin, Y. C. Lin, T. M. Yan, and H. L. Yu, Corrections to chiral dynamics of heavy hadrons: SU(3) symmetry breaking, Phys. Rev. D 49, 5857 (1994).

[37] P. L. Cho and H. Georgi, Electromagnetic interactions in heavy hadron chiral theory, Phys. Lett. B 296, 408 (1992).

[38] P. L. Cho, Chiral perturbation theory for hadrons containing a heavy quark: The sequel, Phys. Lett. B 285, 145 (1992).

[39] N. Jiang, X. L. Chen, and S. L. Zhu, Electromagnetic decays of the charmed and bottom baryons in chiral perturbation theory, Phys. Rev. D 92, 054017 (2015).

[40] L. Meng, G. J. Wang, C. Z. Leng, Z. W. Liu, and S. L. Zhu, Magnetic moments of the spin- $\frac{3}{2}$ singly heavy baryons, Phys. Rev. D 98, 094013 (2018).

[41] G. J. Wang, L. Meng, and S. L. Zhu, Radiative decays of the singly heavy baryons in chiral perturbation theory, Phys. Rev. D 99, 034021 (2019).

[42] M. B. Wise, Chiral perturbation theory for hadrons containing a heavy quark, Phys. Rev. D 45, R2188 (1992).

[43] A. V. Manohar and M. B. Wise, Heavy quark physics, Cambridge Monogr. Part. Phys., Nucl. Phys., Cosmol. 10, 1 (2000).

[44] C. Ordonez, L. Ray, and U. van Kolck, The two nucleon potential from chiral Lagrangians, Phys. Rev. C 53, 2086 (1996).

[45] E. Epelbaum, W. Gloeckle, and U. G. Meißner, Nuclear forces from chiral Lagrangians using the method of unitary transformation. 2. The two nucleon system, Nucl. Phys. A671, 295 (2000).

[46] E. Epelbaum, H. Krebs, and U. G. Meißner, Improved chiral nucleon-nucleon potential up to next-to-next-to-next-toleading order, Eur. Phys. J. A 51, 53 (2015).

[47] D. R. Entem and R. Machleidt, Accurate charge dependent nucleon-nucleon potential at fourth order of chiral perturbation theory, Phys. Rev. C 68, 041001 (2003).

[48] L. Meng, B. Wang, and S. L. Zhu, $\Sigma_{c} N$ interaction in chiral perturbation theory, arXiv:1912.09661. 
[49] F. Z. Peng, M. Z. Liu, Y. W. Pan, M. S. Sánchez, and M. P. Valderrama, Five-flavor pentaquarks and other light- and heavy-flavor symmetry partners of the LHCb hidden-charm pentaquark, arXiv:1907.05322.

[50] Z. C. Yang, Z. F. Sun, J. He, X. Liu, and S. L. Zhu, The possible hidden-charm molecular baryons composed of anticharmed meson and charmed baryon, Chin. Phys. C 36, 6 (2012).

[51] W. L. Wang, F. Huang, Z. Y. Zhang, and B. S. Zou, $\Sigma_{c} \bar{D}$ and $\Lambda_{c} \bar{D}$ states in a chiral quark model, Phys. Rev. C 84, 015203 (2011).
[52] C. W. Xiao, J. Nieves, and E. Oset, Prediction of hidden charm strange molecular baryon states with heavy quark spin symmetry, Phys. Lett. B 799, 135051 (2019).

[53] B. Wang, B. Yang, L. Meng, and S. L. Zhu, Radiative transitions and magnetic moments of the charmed and bottom vector mesons in chiral perturbation theory, Phys. Rev. D 100, 016019 (2019).

[54] E. Epelbaum, U. G. Meissner, W. Gloeckle, and C. Elster, Resonance saturation for four nucleon operators, Phys. Rev. C 65, 044001 (2002). 